\title{
MOEME: Real-Time Mobility and Orientation Estimation for Mobile Environments
}

\author{
Shiraz Qayyum ${ }^{1}$, Umair Sadiq ${ }^{2}$, Mohan Kumar ${ }^{1}$ \\ sq8010@rit.edu,usadiq@nvidia.com,mjkvcs@rit.edu
}

\begin{abstract}
Knowledge of user movement in mobile environments paves the way for intelligent resource allocation and event scheduling for a variety of applications. Existing schemes for estimating user mobility are limited in their scope as they rely on repetitive patterns of user movement. Such patterns neither exist not easy to recognize in soft-real time, in open environments such as parks, malls, or streets. We propose a novel scheme for Real-time Mobility and Orientation Estimation for Mobile Environments (MOEME). MOEME employs the concept of temporal distances and uses logistic regression to make real time estimations about user movement. MOEME is also used to make predictions about the absolute orientation of users. MOEME relies only on opportunistic message exchange and is fully distributed, scalable, and requires neither a central infrastructure nor Global Positioning System. MOEME has been tested on real world and synthetic mobility traces - makes predictions about direction and count of users with up to $90 \%$ accuracy, enhances successful video downloads in shared environments.

Keywords: Opportunistic networks, Mobility estimation, Temporal distance, Logistic regression
\end{abstract}

\section{Introduction}

Resource rich ubiquitous smart phones and wireless communication technologies can be utilized to create an effective network medium for sharing information with

\footnotetext{
${ }^{1}$ Golisano College of Computing and Information Sciences - Rochester Institute of Technology

${ }^{2}$ Nvidia
}

Preprint submitted to Journal of $\mathrm{BT}_{E} X$ Templates

April 7, 2015

(C) 2015. This manuscript version is made available under the Elsevier user license http://www.elsevier.com/open-access/userlicense/1.0/ 
or without infrastructure support. In the wake of growing mobile data traffic, with 70\% rise just in 2012 alone [1], opportunistic networks are an attractive alternative to conventional cellular network.

As mobile environments rely primarily on user mobility as a mechanism for transporting content and data in general, identifying and modeling user mobility provides researchers and network designers with key insights for improving performance, efficiency and productivity. For example, epidemic routing [2] guarantees shortest latencies in message delivery. However, knowledge of user mobility and diffusion has been exploited by various schemes to considerably cut down energy expended in already resource constrained devices with a small tradeoff on delivery times [3][4][5]. With the potential of similar conceivable advantages there are several works which focus on mathematically modeling the human mobility patterns [6]. In another work, researchers characterize pause times, inter-contact times and user mobility speeds based on real world traces collected over different periods of time [7]. Recently, Pajevic et al [8], modeled user mobility in small areas to create building blocks to build a complex queuing model. Communication in these small areas was assumed to be unaffected by user mobility. However, existing schemes that estimate mobility rely on repetitive user patterns and user trace data. Information about trajectories and times of user movement are critical. Moreover, if users deviate from regular patterns of their movement or if no such patterns exist, which is very common in open environments such as parks, malls or streets, the models start falling apart, thereby leading to a serious limitation in guaranteeing accuracy.

To this end, we present a novel scheme called MOEME: Real-Time Mobility and Orientation Estimation for Mobile Environments. To the best of our knowledge, this is a unique contribution to estimate mobility of users in mobile environments in real-time without i) a priori knowledge of user movement patterns, ii) a Global Positioning System and iii) infrastructure support. The key factor which allows MOEME to perform well lies in its message exchange mechanism. MOEME employs the concept of temporal distances - devices exchange information about their temporal distance to other devices. We show that gathering this information for users in real-time is easy and incurs small space complexity. Coupling this information with the model learned us- 
ing logistic regression, MOEME powered devices are able to make estimations about user mobility in real-time. Furthermore, we see that model learned for MOEME generalizes well and is tested to make accurate estimations across both real-world and synthetic traces in the presence of haphazard and random user movements.

MOEME empowers both users and system architects with the knowledge of user mobility. MOEME estimates relative directional mobilities of all the users in a mobile environment, in addition to counting the number of users present within a desired spatiotemporal radius. MOEME can also be employed to predict the absolute orientation of the users in a mobile environment. We demonstrate how MOEME can be used in a variety of scheduling and resource allocation applications. For example, in our recent work we predict the 'contact volume' i.e., the maximum amount of transferrable data between two opportunistically meeting mobile nodes [9]. The accuracy of predicting contact volume can be improved directly with the knowledge of directional mobility of users. Furthermore, it helps in predicting the total volume of information that can be pushed from one end of the network to the other or from a particular source node to a desired destination node.

The novelty of MOEME lies in its ability to estimate user mobilities with no $a$ priori knowledge of movement histories. MOEME can be employed in indoor as well as outdoor environments. MOEME is fully distributed, lightweight and has a time complexity of $O(n)$ at each node, where $n$ is the number of nodes present in the mobile environment. Moreover, MOEME does not rely on Global Positioning System (GPS) or other location tracking systems [10][11], which may be power hungry and thus limit the practicality of the technique.

In our previous work [12], we devised a distributed scheme to estimate the relative directional mobilities and the number of users in a desired spatiotemporal region. Whereas, in this paper we have refined the scheme significantly to estimate the absolute orientation of users in mobile environments.

\subsection{Contributions}

Major contributions of MOEME include: 
1. Estimation of relative directional mobilities of all users in real-time without requiring their movement histories.

2. Estimation of the number of users, that are likely to be within a spatiotemporal region of interest.

3. Predict the absolute orientation of the users in a mobile environment.

To the best of our knowledge MOEME is the first scheme to make the above mentioned contributions for estimations of user mobilities in mobile environments.

MOEME estimates distance of users and number of users within a distance of $300 \mathrm{~m}$ with an accuracy of $89 \%$. The estimates of direction of users are $77 \%$ accurate for nodes within $200 \mathrm{~m}$.

\section{Related Work}

In dynamic and pervasive networks, a significant amount of research has focused on efficient routing schemes [20][21][22][23][24] and content dissemination frameworks [25][26] that exploit repetitive patterns in human movement. Recently, there has been some focus on content and service distributions in open environments, such as parks, malls etc. where history from past visits is not available [3][27] or repetitive patterns do not exist at the time scale of few minutes ( 0 to 15 minutes). There is only one work [28] that aims to create plausible mobility merely through user contacts but requires a centralized server to keep track of all contacts in real time. In contrast, we present the first work that estimates 1) relative directional mobilities of all users in real-time without requiring their movement histories and 2) the number of users, that are likely to be within a spatiotemporal region of interest, through opportunistic contacts in a completely decentralized manner.

\section{System Description}

\subsection{Types of nodes}

We represent the set of all nodes in the pervasive environment by $V=\left\{v_{1}, v_{2}, v_{3}, \ldots, v_{n}\right\}$. Among these $n$, there are $k$ nodes of interest (NOI). We represent the set of NOIs by 


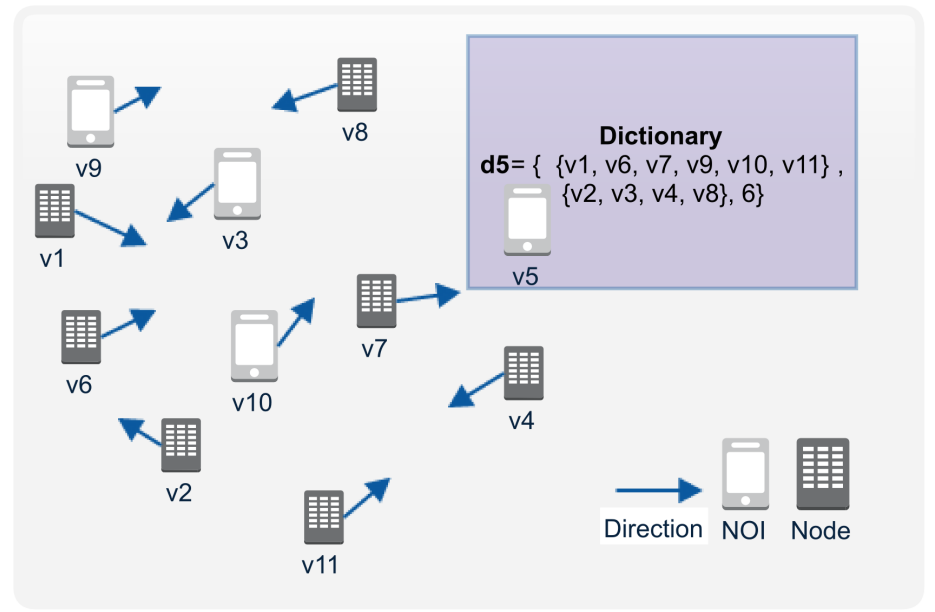

Figure 1: A mobile environment

$P$, where $P \subseteq V$. These nodes of interests are used to make predictions about the direction of motion for the rest of the nodes in the system. The nodes of interests can be static or mobile. Each $v_{i} \in P$ makes a prediction for the nodes in the set $V-\left\{v_{i}\right\}$, the set of all nodes except $v_{i}$.

\subsection{Dictionaries at NOIs}

Every node $v_{i} \in P$, maintains a dictionary $d_{i}$ to keep track of the following information in real-time:

1. Set of nodes moving closer: $C$,

2. Set of nodes moving away: $A$, and

3. Number of nodes moving closer: $\mu$.

The structure of the dictionary is $d_{i}=\{C, A, \mu\}$, where $C \subseteq V, A \subseteq V$ and $C \cup A=$ $V-\left\{v_{i}\right\}$

The Figure 1 depicts a scenario with several regular and monitor nodes. It also shows the contents of dictionary maintained by $v_{5} \in P$. 


\subsection{Underlying network}

The underlying network on which MOEME is built on is inherently opportunistic [13]. The network may comprise of a mix of both static and mobile nodes. However, only the nodes that are within each other's communication range can exchange messages. MOEME uses the information it acquires from other nodes, for local and real-time computation of directional mobilities of other nodes in the environment.

\subsection{Temporal distance}

To keep track of an approximate measure of distance among nodes in a network, the notion of temporal distance is used [3]. It also gives a measure of how fast information can travel among nodes by means of transitive connections among them. At each time instant $t$, every node in the network maintains a timer (Section 3.5) value for the rest of the nodes in the network. The timer values give the measure of temporal distance. However, timer values are not symmetric between nodes due to their distributed nature. It is possible that at some time instance $t_{0}$, the timer value that node $v_{i}$ records for $v_{j}$ is different from the one $v_{j}$ holds for $v_{i}$.

\subsection{Timer update}

Let $t_{v_{i}}\left(v_{k}\right)$ denote the time elapsed since node $v_{i}$ made contact with node $v_{k}$, where $t_{v_{i}}\left(v_{i}\right)=0$. Local timer values for each node are incremented after every time unit, (e.g., 30s, $60 \mathrm{~s}$ etc. depending on devices). When two nodes $v_{i}, v_{j}$ come into contact (within transmission range), $v_{j}$ updates its timer values according to the following rule: $\forall v_{k} \neq v_{j}: t_{v_{i}}\left(v_{k}\right)<t_{v_{j}}\left(v_{k}\right)-t_{a v}$, set,

$$
t_{v_{j}}\left(v_{k}\right)=t_{v_{i}}\left(v_{k}\right)+t_{a v}
$$

where $t_{a v}$ is the measure of distance between two nodes when they are within each others' transmission range. Every node performs this update when it comes into contact with another node. The value of $t_{a v}$ is a small constant greater than zero but less than or equal to one time unit (increment by which local timers are updated) i.e. $t_{a v} \in$ $(0,1]$. Note that it is a different definition of $t_{a v}$ from that in [14] where the value of average time required to travel between the two nodes is included. A small value of $t_{a v}$ 


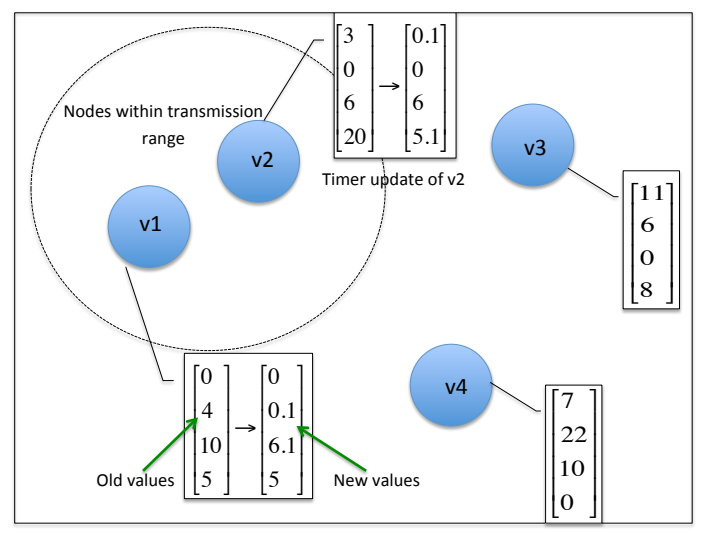

Figure 2: Timer updates

is used as the physical distance between the connected nodes is of little importance. $t_{a v}$ captures the concept of nodes being in contact through other nodes in between. Therefore, value of $t_{a v}>0$ creates a gradient in a connected subset of nodes. Figure 2 shows an example of how the timer values are updated according to rule 1 , when two nodes come within each others' transmission range. In this case, the network comprises of four nodes. Each node maintains timer values for itself $t_{v_{i}}\left(v_{i}\right)=0$, and the nodes in the rest of the network. $t_{a v}$, is set to be equal to 0.1 for the purpose of illustration. The timer values at each node $v_{i}$, where $i=1,2,3$ and 4 are shown in a vector of the form $\left[\begin{array}{l}t_{v_{i}}\left(v_{1}\right) \\ t_{v_{i}}\left(v_{2}\right) \\ t_{v_{i}}\left(v_{3}\right) \\ t_{v_{i}}\left(v_{4}\right)\end{array}\right]$.

\subsection{Additional timers at the time of contact}

MOEME also employs two additional arrays of timers MT and RT, at each node $v_{i} \in V$. Both the arrays contain information that depicts the view of the network each $v_{i}$ observes, just before the time of contact with another node $v_{j} \in V-v_{i}$.

1. MT records the values of all the timers that node $v_{i}$ has for all the other nodes in the network, just before the time of contact. 
2. RT contains the values of timers that some other node $v_{j}$ brings along with it for all the other nodes in the network, just before contacting $v_{i}$.

At the time of contact, first the values in RT and MT are updated and thereafter, the values for regular timers defined in section 3.5 are refreshed according to the update rule 1 . Both these arrays support the usual indexing operation, where the index represents the identity of a particular node, e.g. MT[3] shall access the value in MT for node number 3.

\subsection{Time since last contact and threshold parameter matrix}

Each of the nodes $v_{i} \in V$ record the time elapsed $t_{l}$, since it last contacted some other node in the network. When a node $v_{i}$ comes into contact with another node $v_{j}$, $t_{l}$ is set to zero for both the respective nodes. Hereafter, the value of $t_{l}$ keeps climbing with incremental time units until another contact occurs for a node.

Each of the nodes also has a copy of threshold parameter matrix $\Theta$, which is unique to a type of network and is obtained through the machine learning technique, logistic regression. The rows of $\Theta$ are the individual threshold vectors corresponding to each time since last contact, i.e. integers values of $t_{l} \in[0,10]$. The row values of $\Theta$, timer arrays MT and RT, and time since last contact $t_{l}$ are together used by $v_{i} \in P$ to make a real-time prediction about the relative directional motion of the rest of the nodes in the network.

\section{Learning the threshold parameter matrix}

In MOEME, each node $v_{i} \in P$ strives to estimate the relative direction of motion of the other nodes in the network. In order to do this successfully, these NOIs keep a copy of the threshold parameter matrix. MOEME learns this parameter matrix $\Theta$ offline by applying logistic regression on data extracted from mobility traces. The novel aspect of the proposed scheme is the fact that it is completely distributed in nature. NOIs can locally make estimates for the directional mobility of other nodes in the network. There is no centralized agent, thereby making the scheme robust and fault-tolerant. 


\subsection{Logistic regression}

The objective of machine learning algorithm logistic regression, is to produce a threshold parameter vector $\theta$ that minimizes an appropriate cost function $J(\theta)$ for a subset of the given data [15]. The algorithm was run a number of times, each time on a different subset of the original data. The original data is filtered according to the $t_{l}$, the time since last contact values, to obtain subsets of the data, where $t_{l}$ takes integers values in the range $[0,10]$. All the obtained $\theta$ vectors are transposed and stacked together to form the threshold parameter matrix, $\Theta$. At the time of directional mobility estimation MOEME selects an appropriate row from the matrix $\Theta$ based on $t_{l}$ and processes it with the feature vector $x$, where the feature vector is calculated using both MT and RT . Logistic regression serves us well because the problem at hand falls under binary classification problems [16].

\subsubsection{Sigmoid function}

It has been shown that a naive linear hypothesis

$$
h_{\theta}(x)=\theta^{T} x=\theta_{0}+\theta_{1} x_{1}
$$

where,

$$
\theta=\left[\begin{array}{l}
\theta_{0} \\
\theta_{1}
\end{array}\right] \text { and } x=\left[\begin{array}{c}
1 \\
x_{1}
\end{array}\right]
$$

, will work poorly in the case of classification problems, although it works well for linear regression [16]. Therefore, in order to get a better estimate, the sigmoid function is used and the hypothesis is defined as

$$
h_{\theta}(x)=g\left(\theta^{T} x\right)
$$

where,

$$
g(z)=\frac{1}{1+\exp ^{-z}}
$$




\subsubsection{Cost function and gradient}

The data set used comprises two columns, $x_{1}$ and $y$. At each time instance, for each pair of nodes, $x_{1}$ and the corresponding $y$ values are calculated. $y$ can take values either 0 or 1 , where 0 means the nodes in consideration are in reality moving away, whereas 1 means they are moving closer. We use the notation $x^{i}$ and $y^{i}$ to denote the $i^{t h}$ example in the training data set, i.e. the $i^{t h}$ row in the data set. Simply minimizing the sum of the squares of errors, where errors are usually defined as a difference in the actual $y$ and the guessed values, shall not work [16]. Logistic regression algorithm fails to find a suitable global minima as the resulting cost function in that case is not convex. To resolve this problem, a better cost function that is shown to have a global minima is used;

$$
J(\theta)=\sum_{i=1}^{m}\left[-y^{i} \log \left(h_{\theta}\left(x^{i}\right)\right)-\left(1-y^{i}\right) \log \left(1-h_{\theta}\left(x^{i}\right)\right)\right]
$$

and the gradient of the cost is a vector of the same length as $\theta$ where the $j^{\text {th }}$ element for $(j=1,2 \ldots, n)$ is given by

$$
\frac{\partial J(\theta)}{\partial \theta_{j}}=\frac{1}{m} \sum_{i=1}^{m}\left[\left(\left(h_{\theta}\left(x^{i}\right)\right)-y^{i}\right)\left(x_{j}^{i}\right)\right]
$$

The cost function in Equation 6 captures the notion of penalizing the learning algorithm when it makes a wrong prediction. Consider the case when $y=1$ in the learning example and the hypothesis outputs $h_{\theta}(x)=0$, thereby making a wrong prediction. Therefore, the term $-\log \left(h_{\theta}(x)\right.$ in the cost function will be $-\log (0)$, which approaches infinity. However, contrary to this, if the hypothesis produces $h_{\theta}(x)=1$, which is equal to the original $y=1$ label, then the cost drops down to zero. The same reasoning can be applied to show the desired behavior of the cost function when $y=0$.

Execution of the algorithm, on a subset of the data a threshold parameter vector $\theta$ is obtained which minimizes the above mentioned cost function $J(\theta)$. All such threshold parameters obtained from different subsets of data form the threshold parameter matrix $\Theta$. 


\subsection{Feature selection}

The choice of features in any machine learning algorithm play a pivotal role in the overall performance and accuracy of learning. We carefully selected the following features to use:

1. Time since last contact, $t_{l}$;

2. Node's timer value for the node in consideration, $v_{i}\left(v_{k}\right)$; and

3. Contacting node's timer value for the node in consideration, $v_{j}\left(v_{k}\right)$.

The rationale for using $v_{i}\left(v_{k}\right)$ and $v_{j}\left(v_{k}\right)$ is the fact that the difference of these two values gives us an indication whether the node in consideration is traveling towards or away from $v_{i}$. If $v_{i}$ encounters a contacting node $v_{j}$ which has a lower timer value for the node in consideration $v_{k}$, then intuitively $v_{i}$ should be moving closer to $v_{k}$. However, if $v_{j}$ 's timer is higher, then for most of the times an opposite inference can be made. We also make use of $t_{l}$, though this feature is not fed into logistic regression directly. We primarily use it to filter the data and run the algorithm on a subset of the data. It has been observed that the difference, $v_{i}\left(v_{k}\right)-v_{j}\left(v_{k}\right)$, which forms the column values $x_{1}$, contains more accurate information when $t_{l}$ was small. This fact is also intuitive because with the passage of time the information becomes stale and less reliable.

\section{Real-time estimation of directional mobility}

\subsection{Estimation at NOIs}

As the nodes in the network move around and come into contact with each other opportunistically, they exchange information about their timers and update MT and RT. When a node $v_{i} \in P$ wishes to estimate the relative directional mobilities of the rest of the nodes in the network it first calculates the difference of arrays,

$$
\Delta T_{i}=M T_{i}-R T_{i}
$$

that it maintains. The subscript $i$ denotes that the above expression is evaluated for the $i^{\text {th }}$ node. The node then checks its $t_{l}$ value, i.e., how long ago did $v_{i}$ make its 
last contact with some other node in the network. Based on the current $t_{l}$ value, $v_{i}$ chooses the corresponding $t_{l}^{t h}$ row $\left(\theta^{T}\right)$ from the threshold parameter matrix $\Theta$. Now if $v_{i}$ wishes to make a prediction about the direction of motion for node $v_{j}$, it calculates the following:

$$
z=\theta^{T} \times\left[\begin{array}{c}
1 \\
\Delta \mathbf{T}_{\mathbf{i}}[\mathbf{j}]
\end{array}\right]
$$

The index $j$ fetches the $\boldsymbol{\Delta} \mathbf{T}_{\mathbf{i}}$ value for $v_{j}$. Subsequently, this $z$ value is used in Equation (5) to get $g(z)$. If $g(z)$ is greater than or equal to zero, node $v_{i}$ estimates the direction for $v_{j}$ as approaching closer, whereas, $g(z)$ less than zero would mean otherwise. MOEME repeats the above mentioned procedure for each $v_{i} \in V-\left\{v_{i}\right\}$.

\subsection{Time complexity and scalability}

MOEME is extremely lightweight in terms of computation. The processing that a node $v_{i} \in P$ has to do to estimate the direction of motion of another node $v_{j} \in$ $V-\left\{v_{i}\right\}$, comprises a fixed number of computational steps. The first step is simply taking the difference of two values to obtain a real number: $\Delta \mathbf{T}_{\mathbf{i}}[\mathbf{j}]$. The computation in Equation (9) is a multiplication of two $2 \times 1$ vectors, where the elements of the vectors are in $\mathbb{R}$. Finally, the computation of Equation $(5)$ is also a fixed number of arithmetic operations. In order for a node to compute the direction for all the other nodes, the above mentioned steps shall be repeated $n-1$ times, where $n$ is the number of nodes in the environment. Therefore, the time complexity of MOEME for real-time estimation of directional mobilities is $O(n)$.

The machine learning algorithm used in this paper is lightweight and is essentially an offline training algorithm, which can run on a server machine. Therefore it does not affect real-time performance of MOEME. The machine learning algorithm we use is fairly quick and is able to train a data set with e.g., $18 \times 18 \times 90 \times 2=58320$ $($ nodes $\times$ nodes $\times$ trace_duration_in_minutes $\times 60 / 30)$ rows in under 3 minutes on a $2.4 \mathrm{GHz}$ processor and $2 \mathrm{~GB}$ RAM, to produce a resultant threshold parameter matrix.

This analysis shows that MOEME can run with blazing fast performance on present day smart phones, that usually have processor speeds on the order of GHz. Therefore, 
MOEME is scalable to mobile environments consisting of nodes on the order of hundreds.

\section{Direction estimation with orientation sensor}

Thus far we have described a methodology to predict the directional mobility and count of nodes in a spatiotemporal region. However, the directional mobility is limited to estimating whether the nodes in question are moving towards or away from a node of interest. In this section, we detail the use of the orientation sensor on smartphone devices to get additional information about the angle of movement of a node.

\subsection{Local orientation readings on a device}

Modern smartphones are equipped with orientation sensors. For example, an Android device can make use of getOrientation in the SensorManager API [17] to get information about its orientation. In this paper, every node $v_{i}$ maintains a local orientation reading, which is essentially its own bearing value measured with respect to the magnetic north. We use the bearing values rounded to the nearest integer and are in the range $[0,360)$. The value 0 corresponds to the the true magnetic north and the bearing increases when the device rotates eastward (clockwise). Figure 3 depicts the orientation coordinate system used in this paper.

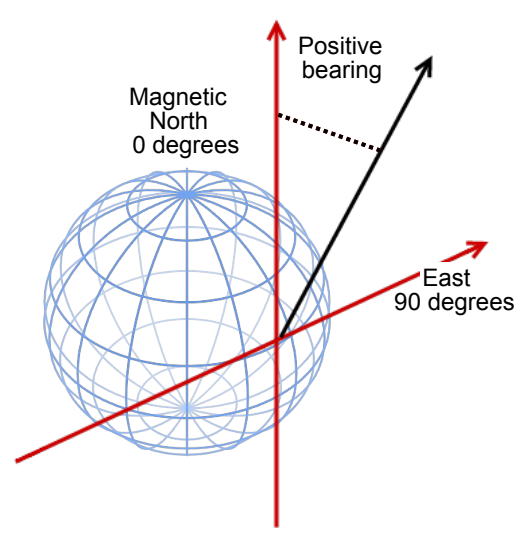

Figure 3: Orientation coordinate system 


\subsection{Sharing of orientation information}

In a mobile environment the nodes move and often make contact with other nodes when they come within each other's radio communication range. A contact is an event where the nodes may exchange relevant information that can potentially help in achieving some goal. In this paper, we use the notation $b_{v_{i}}\left(v_{j}\right)$ to denote the bearing value that node $v_{i}$ maintains for node $v_{j}$. In other words, it is the absolute orientation of node $v_{j}$, perceived by node $v_{i}$. When the two nodes $v_{i}$ and $v_{j}$ come within each other's communication range, they inform the meeting node about their own orientation, i.e., the local bearing reading $b_{v_{i}}\left(v_{i}\right)$ at $v_{i}$ is given to node $v_{j}$ and vice versa. Moreover, much like the discussion in Section 3.5, each node maintains orientation information not just about itself or the meeting node, but about every other node in the network.

Initially, every node $v_{i} \in V$ in the network starts by keeping track of its own local bearing value using the orientation sensor. The bearing values for all other nodes at this node are initialized to -1 , which means they are irrelevant and cannot be trusted. During the course of time, when the nodes move and a node $v_{i} \in V$ makes contact with $v_{j} \in V$, then the node $v_{j}$ updates the orientation values according to the following bearing update rule:

$$
\begin{aligned}
\forall v_{k} \neq v_{j}: t_{v_{i}}\left(v_{k}\right)<t_{v_{j}}\left(v_{k}\right)-t_{a v}, \text { set, } & \\
& b_{v_{j}}\left(v_{k}\right)=b_{v_{i}}\left(v_{k}\right)
\end{aligned}
$$

The above update rule means that a node will prefer more recent perspective of orientation values that may be brought to it by other nodes in the network.

\subsection{Prediction criterion and features}

As time progresses, the nodes in the network share timer and orientation information among themselves, it is of interest to know whether the orientation information they maintain about other nodes is likely to be less accurate and hence a lower trust value. Intuitively, if the orientation information was updated long ago, then it should not be trusted. However, we relax the prediction criterion and instead of predicting the exact orientation angle of the nodes, we will tolerate the error within bounds.

In order to understand the scope of prediction consider the following function, 


$$
Q(x)=\left\{\begin{array}{lll}
1 & \text { if } & 0 \leq x<90 \\
2 & \text { if } & 90 \leq x<180 \\
3 & \text { if } & 180 \leq x<270 \\
4 & \text { if } & 270 \leq x<360
\end{array}\right.
$$

that maps the integers between the range $[0,360)$ to four different quadrants(divisions). Now, suppose the predicted bearing value at a node $v_{i}$ for $v_{j}$ is $b_{\text {predict }}\left(v_{j}\right)$ and the actual bearing value of $v_{j}$ is $b_{\text {actual }}\left(v_{j}\right)$, at some time. Then, if $Q\left(b_{\text {predict }}\left(v_{j}\right)\right)=$ $Q\left(b_{\text {actual }}\left(v_{j}\right)\right)$, then we term the prediction as correct. In other words, it means if the predicted value and the actual value fall in the same division then the prediction is deemed correct. Note that, a different prediction criterion can easily be chosen by using a function $Q(x)$ with a different (smaller or greater) number of divisions. In this paper, we have chosen four divisions as they naturally correspond to the quadrants. Moreover, note that it is not necessarily a very tolerant criterion, as the bearing values close to the boundaries i.e., $0,90,270$ etc. may deviate only by a few degrees, and result in a wrong prediction.

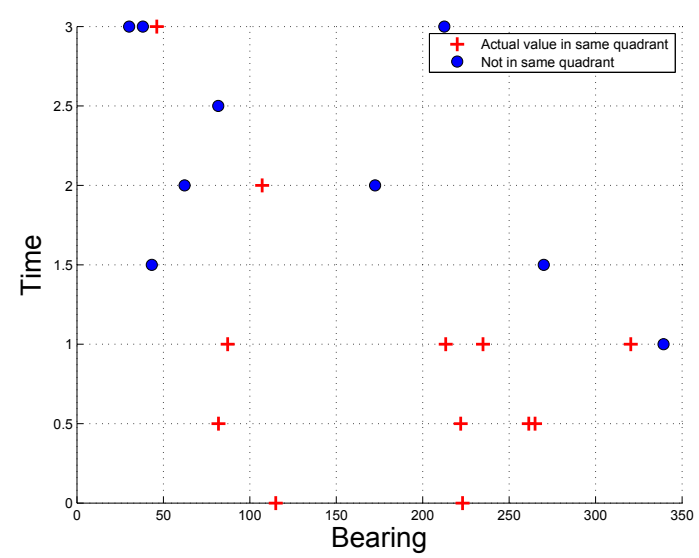

Figure 4: Data at an arbitrary node and time showing whether the orientation values fall within the same quadrant/division

To make predictions about the orientation of another node $v_{j}$, a node $v_{i}$ uses the 
following two features as input to the sigmoid function:

1. Timer value for node $v_{j}$; and

2. Bearing value for node $v_{j}$.

While training the data set the actual bearing values of the nodes are plotted against the aforementioned features and a decision boundary is computed. The decision boundary can later be used to make predictions about the orientation of the nodes in real-time. Figure 4 shows one such data set at a node that was obtained during the training process. It shows whether the orientation values at a node fall within the same division. It can be seen that for higher timer values the actual value of the bearing is likely to move out of the same quadrant/division.

\section{Implementation}

The architecture depicted in Figure 5 can be broken down into three major parts, i.e. Wi-Fi Direct, Network and Estimation, wherein each module is responsible to carry out a specific group of related tasks. MOEME is implemented and tested on Google Galaxy Nexus phones with a 1.2 GHz dual-core ARM Cortex-A9 CPU and 1 GB of memory. The phones are powered by Android 4.3 Jelly Bean.

\subsection{Wi-Fi Direct module}

Wi-Fi Direct lets mobile devices connect with each other directly without the need of an intermediate access point to over a 100m. The technology is available in Android versions greater than 4.0 and is supported by the above mentioned phones we use for implementing MOEME.

As part of MOEME the Wi-Fi Direct module is responsible for carrying out tasks related to establishing the connectivity with other nearby devices. Wi-Fi manager first tries to discover nearby peers by running a separate thread of execution. The interval between successive discovery initiations can be tweaked based on the environment, where a crowded environment would call for discovering often. Once the discovery returns a list of nearby devices, MOEME tries to connect to up to four available devices in the surrounding. This limitation on the design is placed to keep the implementation 


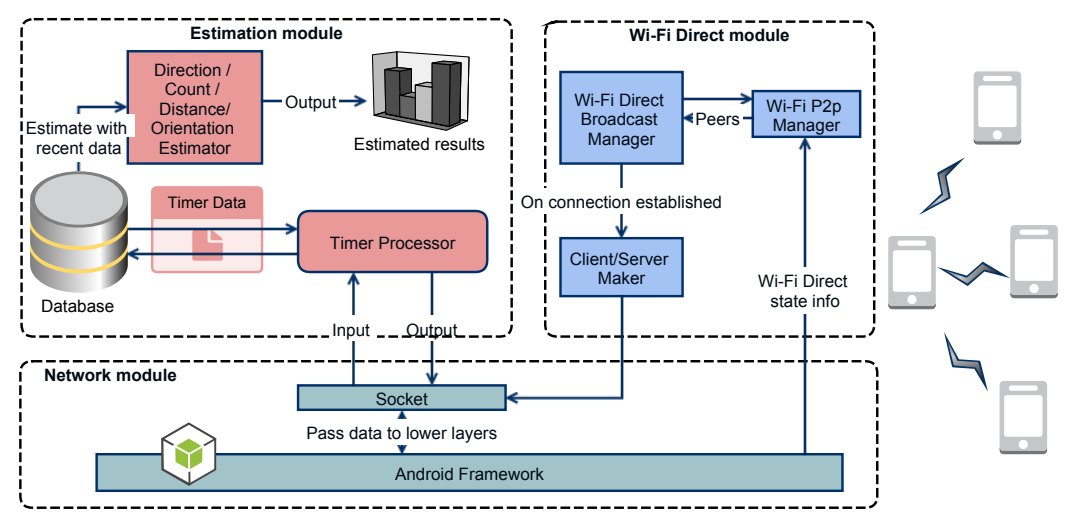

Figure 5: Architecture of MOEME implementation on an Android framework

more responsive and agile. However, this does not mean MOEME misses out a chance to connect to other devices. Once the timer values are exchanged between the current set of connected devices, MOEME moves forward and connects to a set of mutually exclusive devices the next time around. Once there are no new devices to exchange timers with, a device cycles back to connect with the first set of nearby peers, if any or all of them are still available.

The information about connection setup or a disconnect is relayed via Wi-Fi Direct broadcast manager over to client/server maker. As the name suggests this sub-module is responsible for opening up server or client sockets depending on whether the connection earlier resulted in making a device a 'group owner' or not respectively. A group owner essentially acts as a hub, thereby routing messages among a peer to peer group. Therefore, a group owner is responsible for running and terminating several worker threads, each of which talks with the respective client device. On the contrary, each of the client devices simply connect to the group owner and synchronize their local timer values.

\subsection{Network module}

The network module in Figure 5 shows the lower level networking layer. Sockets are essentially pipes between the estimation module and the Android framework, that carry streams of information wrapped around by conventional buffered readers and writers. The Android framework takes care of dealing with the low level networking i.e., sending and receiving the actual bits of information. Apart from this, the framework also monitors the state of several other ongoing processes, such as, peer listening 
and connection calls.

\subsection{Estimation module}

The primary purpose of MOEME is to estimate mobilities of users in the surrounding environment. The estimation module, contains implementation of the logic described earlier in timer update rule and real-time estimation of orientation/directional user mobilities. First, timer processor receives new information about a peer's timer values. MT and RT are updated along with the orientation information, in an SQLite database. Subsequently, a node's own timer values and local orientation information are pulled out from the database and transferred over to the connected peer. Subsequently, the timer values and orientation information are updated according to the timer update rule 1 and bearing update rule 10 respectively.

Finally, the mobility estimator reads the most current view of the network and estimates the directional mobilities and orientation of the users it has information about. The results of the estimation are then presented to the user in the form of Android activities. Which are basically a way for the user to interact with an Android UI. Android makes it easy for several applications to communicate with each other. Therefore, it is possible for other applications to use services of MOEME, given the correct privileges are in place.

\section{Simulation and Analysis}

Performance of prediction is evaluated for estimating direction, distance, and count of nodes within a certain region. The performance varies when there has been a long time since last contact (represented by time range) and when nodes are located physically further apart (represented by distance range). Extensive simulations are run on real as well as synthetic mobility traces. The real mobility trace has been collected from participants that carry GPS receivers which log position at 30 second intervals. These traces are collected in five different environments, but we show representative results from a State Fair [18] in Figures 6 and 7. State Fair's area is 500m x 500m and the nodes have typical walking speeds with recurrent pauses at different stalls. 
In order to make a comparison with suitable number of nodes, track logs from each day are considered to be that of a separate user. These logs have durations from around one to twelve hours each day. We truncate all $\operatorname{logs}$ to 90 minutes during which 18 user devices record their location. As the trace logs have different durations, ranging from one hour to twelve hours, the logs were clipped to make the analysis consistent, i.e., for the first 90 minutes. For longer than 90 minutes, the number of nodes that log information for a longer duration decreases sharply. So we decided to use 90 minute duration with $18 \operatorname{logs}$ available (instead of e.g. 4 hours duration with only $7 \operatorname{logs}$ available for that duration).

Similar preprocessing steps were performed on the other four real-world mobility traces. We show the orientation prediction accuracy of MOEME for all of them in Figure 8 . Table 1 shows the number of nodes along with the duration to which each trace was clipped for uniformity.

Table 1: Real-world mobility traces used in this paper to show orientation prediction accuracy

\begin{tabular}{c||c||c}
\hline Trace & Number of nodes & Duration (minutes) \\
\hline \hline State Fair & 18 & 90 \\
\hline Orlando & 41 & 131 \\
\hline New York & 39 & 74 \\
\hline NCSU & 35 & 103 \\
\hline KAIST & 92 & 253 \\
\hline
\end{tabular}

Synthetic mobility traces are generated using SLAW mobility model[19] with 20 nodes. Length of each step, and pause duration is different for all nodes throughout the trace duration, where we only specify the exponent of distribution from which a random value is selected for every node at every step. The environment is made further heterogeneous by changing velocity, minimum/maximum pause durations, and minimum step length for two classes of nodes i.e., 10 slow and 10 fast moving nodes. Details about trace settings are provided in Table 2

\subsection{Simulation setup}

Figures 6 and 7 show the average of results at all nodes at different times. Since, the accuracy results belong to a Bernoulli distribution, the $99 \%$ confidence interval are ex- 
Table 2: SLAW mobility trace used for simulation

\begin{tabular}{c||c|c}
\hline \multicolumn{1}{c||}{ Description } & slow & fast \\
\hline \hline Exponent of step length distribution & \multicolumn{2}{|c}{1.6} \\
\hline Exponent of pause time distribution & \multicolumn{2}{|c}{0.6} \\
\hline Hurst Parameter & \multicolumn{2}{|c}{0.75} \\
\hline Velocity of node & $1 \mathrm{~m} / \mathrm{s}$ & $5 \mathrm{~m} / \mathrm{s}$ \\
\hline Minimum step length & $5 \mathrm{~m}$ & $25 \mathrm{~m}$ \\
\hline Minimum pause duration & $30 \mathrm{~s}$ & $7.5 \mathrm{~s}$ \\
\hline Maximum pause duration & $600 \mathrm{~s}$ & $60 \mathrm{~s}$ \\
\hline Simulation area & \multicolumn{3}{|c}{$500 \times 500 \mathrm{~m}$} \\
\hline
\end{tabular}

tremely close to the average values due to large number of samples (greater than 2000). Therefore, the confidence intervals are not plotted. Parameters used for prediction are $t_{a v}=3 \mathrm{~min}$ and the linear fit line to compute timer thresholds based on distance threshold is distance $=\sqrt{t_{v_{i}}\left(v_{j}\right)}+28$. This line is based on linear fit between square root of timers [3] and actual distances between nodes in the state fair trace. Thus, to estimate if distance between two nodes is less than a specific value, we check the node's timer if it is less than the value satisfied by the above equation ${ }^{3}$ We make predictions for three measures:

1. Direction: relative direction of users with respect to NOI;

2. Distance: distance of a user from NOI to be less than a specific threshold,

3. Count: number of users whose distance from NOI is less than a specific threshold; and

4. Orientation: the absolute bearing value of users predicted at a NOI.

The prediction of above measures is analyzed against two variables: distance range and time range. For example, when distance range is $200 \mathrm{~m}$, it means that the prediction is made for a selection of users that are within $200 \mathrm{~m}$ of any NOI. When time range is $10 \mathrm{~min}$, it means that the prediction is made for a selection of users whose timer value has been updated at the NOI within previous $10 \mathrm{~min}$. Thus, a smaller time range means that prediction is made for a selection of users about whom some information (the

\footnotetext{
${ }^{3}$ The unit of distance and time is in meters and minutes respectively
} 
time value) has been updated recently. Intuitively, prediction for small values of time range and distance is better because it means that the user is situated nearby and the information (time value) about the node has been updated recently.

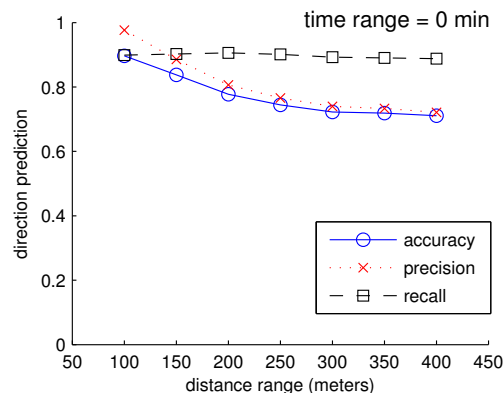

(a) Prediction of direction of nodes that are close is better

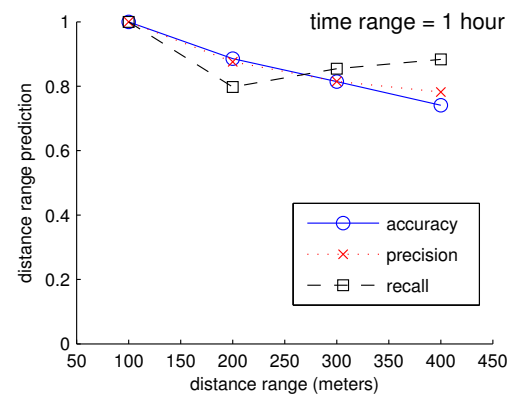

(c) Prediction of distance of nodes that are close is better

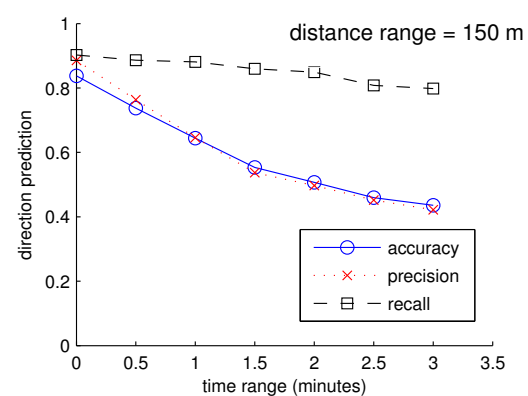

(b) Prediction of direction of nodes decreases sharply when no new information is available in

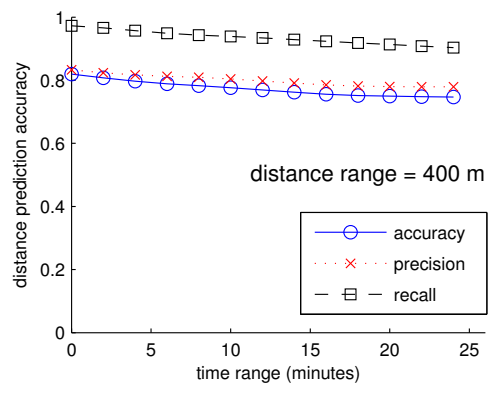

(d) Prediction of distance of nodes decreases moderately when no new information is available in past $10-20$ minutes

Figure 6: Direction and distance estimation

\subsection{Direction estimation}

From the logistic regression model, we find different values of $\theta$ specific to state fair trace for different values of time since last contact. In order to generalize, we find that use of $\theta_{0}=0$ gives good results for both the state fair as well as synthetic mobility models. This simplifies to simply using MT-RT, and inferring the movement of user to 
be moving close to NOI for positive difference in timers. Figure 6c shows the precision, recall and accuracy for all users (i.e. the results are average by considering each user to be the NOI) at different distance ranges. Precision and recall show the efficiency of prediction when a specific user is moving closer to the NOI. The recall is greater than $90 \%$ at all distance ranges, however, precision and accuracy drop from $90 \%$ to $75 \%$ when the distance range increases from $100 \mathrm{~m}$ to $300 \mathrm{~m}$. This is because, for users that are further away, it takes longer for new information to reach a NOI. However, even a $75 \%$ accuracy is extremely useful as there does not exist any other mechanism to estimate direction of users in physical proximity by using the opportunistic transfer of information and without any GPS device.

In contrast to prediction robustness against users that are located further away, time since last contact (represented by time range) sharply decreases the precision and accuracy. This is reflected in Figure 6d, where prediction accuracy drops from 90\% to 50\% in just over 2.5 minutes. This is because there is almost zero correlation in direction of user movement between two instants of time separated by more than 3 minutes. We believe this is a special case of state fair traces and in other environments with directional flow, the prediction accuracy will drop more gradually. However, this effect needs to be tested based on collection of real mobility data from additional environments with directional flows.

\subsection{Distance estimation}

Since, the direction estimation is sensitive to time range, we estimate the distance of users in proximity. Thus, we find whether a particular user is within a specific distance of NOI where this specific distance is given by the distance range. Figure 6 . shows that distance prediction (precision and accuracy) drops from $100 \%$ at $100 \mathrm{~m}$ ( $100 \mathrm{~m}$ is also the transmission range of NOI) to $80 \%$ at $300 \mathrm{~m}$ whereas recall initially decreases to $80 \%$ but then increases back to $90 \%$ at distance range of $400 \mathrm{~m}$. The drop in precision and accuracy is intuitive but the increase in recall is an artifact of specific user mobility in the state fair trace and is a result of linear fit of: i) distance with square root of temporal distance; and ii) the space constraint of approximately 500x500m in state fair. Figure $6 \mathrm{~b}$ shows that distance prediction is only moderately affected by the 
time range i.e. the accuracy drops from 0.80 to 0.77 when time range increases from 0 min to $25 \mathrm{~min}$. The reason is that even though users change direction a lot more rapidly (very likely in 3 min [Figure 6d]), they do not move out of a region that quickly. Thus a user within $400 \mathrm{~m}$ of NOI is likely to remain in the region for another 20 minutes even though it may keep on changing directions more quickly.

MOEME scheme performs better in dense environments. With higher ranges, the latency for receiving timer values is larger in environments such as the ones we are targeting (parks, malls, streets etc.). With longer delays there is a higher probability that the user would change direction thus making it difficult to use the prediction made by MOEME. This is an inherent characteristic of human mobility in some dynamic environments. MOEME would perform better with high range if users do not change direction frequently.

\subsection{Count estimation}

In addition to the distance estimate, we also count the number of users within a certain region as defined by the distance range. Since, this count may not be exact, we test the prediction with different levels of tolerance i.e. a tolerance of 1 means that actual count and predicted count can differ at most by one. Therefore, prediction accuracy is higher when tolerance is 3 as opposed to 1. This is also reflected in Figure 7a. Similar to distance estimation, the count estimation is more robust to time ranges in comparison with distance ranges. When tolerance is 1 , the count prediction drops to $50 \%$ at a distance range of $200 \mathrm{~m}$ - but with tolerance equal to 2 , the accuracy is close to $80 \%$ as shown in Figure $7 \mathrm{a}$.

Figure $7 \mathrm{~b}$ shows the effect of time ranges. For tolerance equal to 1 , the accuracy drops below $50 \%$ at $15 \mathrm{~min}$ but at tolerance equal to 3 , the accuracy stays above $80 \%$ for up to time range of $25 \%$ which is quite good.

In order to analyze the error in count more deeply, Figure $7 \mathrm{c}$ and Figure $7 \mathrm{~d}$ show a histogram of different in actual and predicted count. As seen from the figure, the tolerance of 3 enables an accuracy of up to $80 \%$ in state fair and 70\% in SLAW mobility model. The error in SLAW mobility is more dispersed as it is a random movement model and there is much less correlation in directions of user movement. 


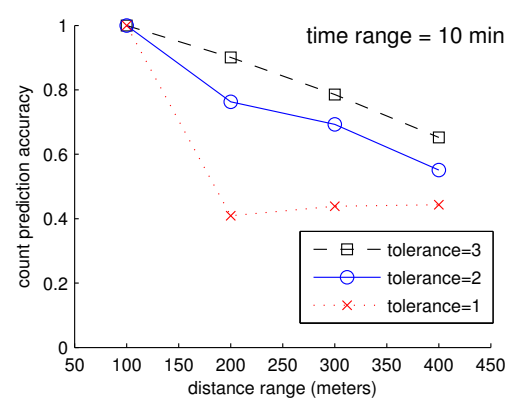

(a) Prediction of count of nodes that are close is better

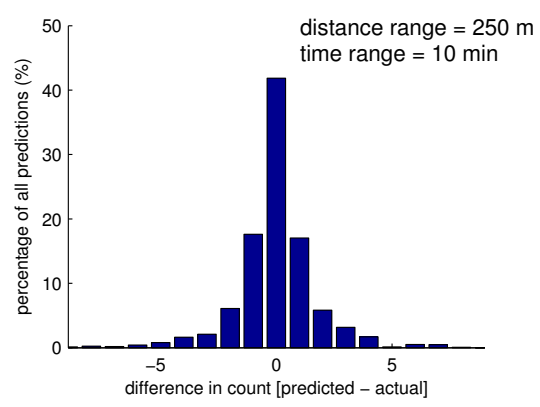

(c) $80 \%$ of count predictions are within an error of a single count in state fair

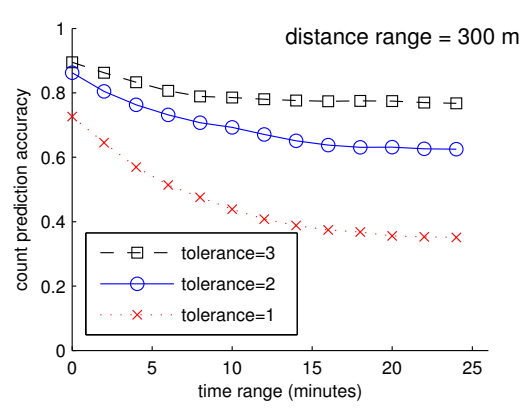

(b) Prediction of count of nodes decreases moderately when no new information is available in past 10-20 minutes

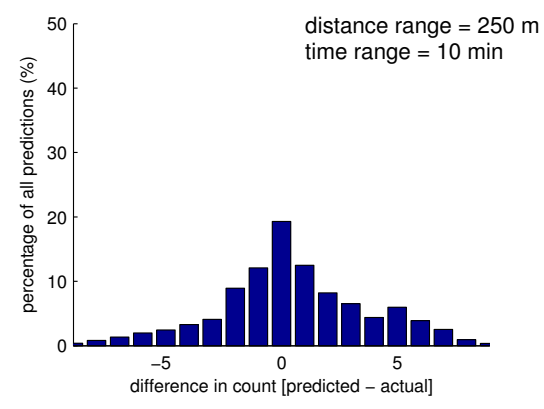

(d) $80 \%$ of count predictions are within an error of a single count in SLAW Mobility Model

Figure 7: Count prediction

\subsection{Orientation estimation}

The orientation of users is predicted at NOIs using the trained logistic regression model. The plot shown in Figure 8 is generated for users in five different real-world mobility traces. The simulation is run 1000 times, and in each run arbitrary NOIs are chosen that make predictions about the orientation of other nodes for the entire duration of the trace. It is observed that the prediction accuracy is $100 \%$ accurate when the timer information is just exchanged. This is because, the bearing values exchanged at the time of contact are fresh and depict accurate information about the actual orientation of nodes. However, the prediction accuracy drops as the temporal distance increases 


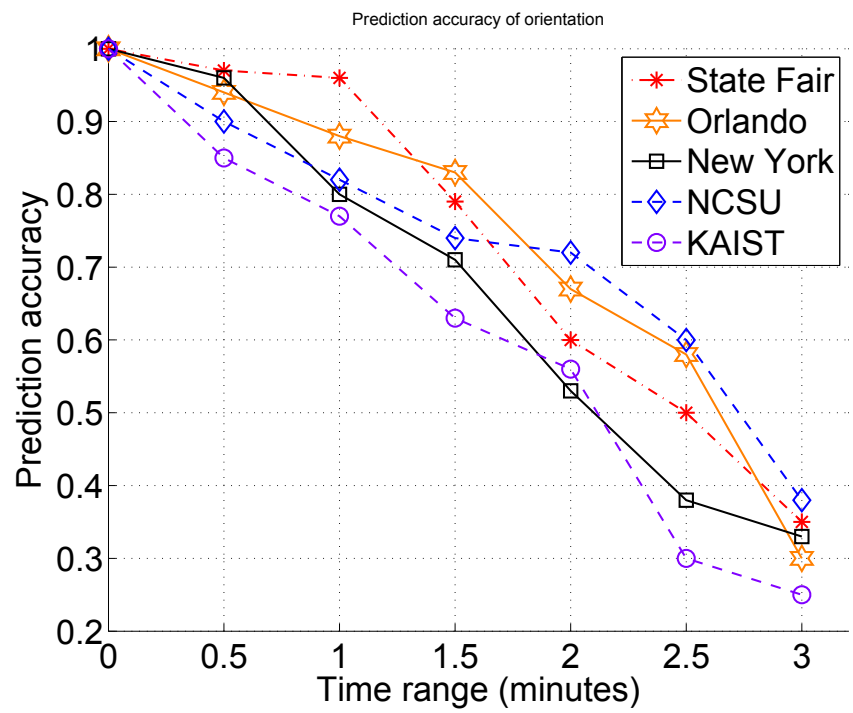

Figure 8: Prediction accuracy of orientation against timer values. The accuracy is plotted for the five different real-world mobility traces.

between the nodes. This means that no new information about the bearing values of the nodes in question is known, hence the prediction accuracy drops sharply. KAIST trace shows the worst prediction accuracy, and we believe it is because it has the maximum number of nodes for which the timer and bearing values are not updated soon enough.

\subsection{Successful downloads in a video sharing application}

In order to show an application of MOEME, a video sharing service is simulated in a mobile environment. At the start of the experiment, a cluster of three nodes is chosen, wherein each node downloads a chunk of video from the cellular network and maintains it for future downloads over speedy and free local Wi-Fi links. Other mobile nodes in the environment can download individual chunks of original video from the provider nodes if they come within communication range and the Wi-Fi link is not broken for the entire duration of the download.

As MOEME powered devices can estimate directional mobilities, a simple heuristic is used to improve performance. The provider nodes in the cluster estimate the direction of movement from each other and keep a memory of past $\lambda$ number of same consecutive direction predictions. Figure 9 shows $\lambda$ varying from 1 to 6 on the $\mathrm{x}$-axis. Provider nodes continuously monitor each other's movement. Suppose a provider node $v_{i}$ is found to move away from the cluster for $\lambda$ consecutive predictions, a suitable cur- 


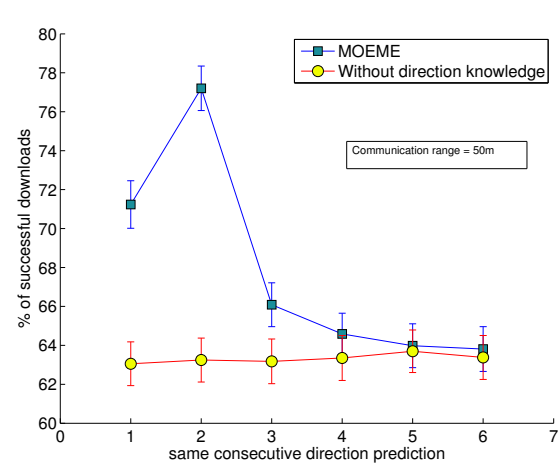

(a) Gain with MOEME is most pronounced for a short communication range $(50 \mathrm{~m})$

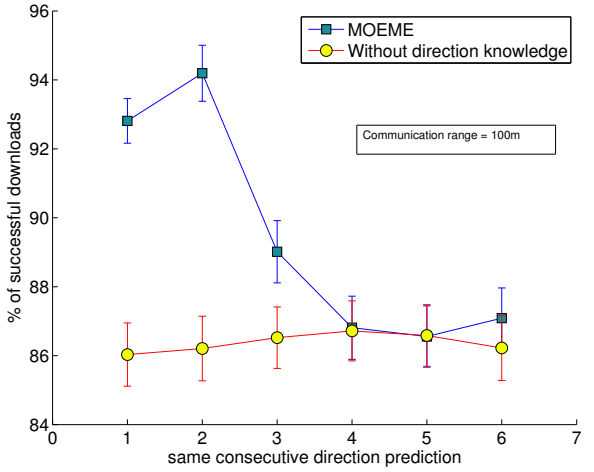

(b) Performance with MOEME peaks at $94 \%$ for $100 \mathrm{~m}$ communication range

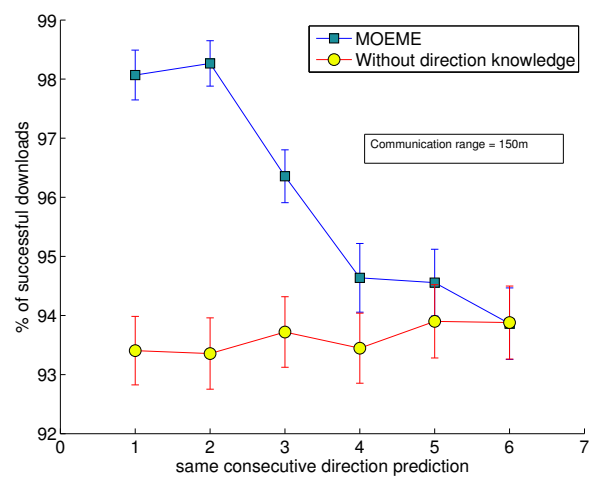

(c) Long communication range $(150 \mathrm{~m}) \mathrm{im}-$ proves overall success rate

Figure 9: Percentage of successful downloads for different communication ranges. MOEME performs better in all three cases 
rent non provider node moving towards the cluster for $\lambda$ consecutive predictions is identified to take over from $v_{i}$ as a new provider. Figure 9 shows the average successful percentage of downloads performed for 2000 runs of simulation. It is observed that, using a simple heuristic based on MOEME, for a communication range of 50 $\mathrm{m}$, average number of downloads served is improved by more than $16 \%$. The gain of MOEME over methods that do not use directional information is most noticeable for short communication ranges. However, it is observed, that the overall performance of servicing video downloads improves for longer communication ranges. This is because, link failures in such an environment are relatively less. Moreover, it is observed that with $\lambda=2$, MOEME performs best and the performance asymptotically degrades with higher $\lambda$. This is because, it is a stricter condition and finding nodes that keep their relative directional movement constant over longer period of times are hard to find in a dynamic mobile environment.

\section{Conclusion}

This paper presents the first fully-distributed and real-time scheme to estimate direction, distance and users in the proximate environment as well as the count of users within a specific distance of any node of interest (NOI). The prediction of direction utilizes a logistic regression model and gives more than $77 \%$ accuracy for nodes within $200 \mathrm{~m}$ of the NOI whenever the NOI's timer is updated for a particular user. The estimates of distance and count are more robust even when no new contact has occurred in the past 10 to 15 minutes and prediction accuracy is greater than $80 \%$ for users with $300 \mathrm{~m}$ of the NOI. The proposed scheme demonstrates similar performance in terms of prediction accuracy for real and synthetic mobility traces. It is also shown that MOEME can be employed to enhance the percentage of successful downloads by over $14 \%$ for a video sharing service in a mobile environment. This opens up possibilities for intelligent resource allocation and event scheduling in various other multimedia applications. 


\section{Acknowledgments}

This work was supported in part by the US National Science Foundation under grants CSR 0834493, and CNS-1150192. Any opinions, findings, and conclusions or recommendations expressed in this paper are those of the authors and do not necessarily reflect the views of the National Science Foundation.

\section{References}

[1] C. V. N. Index, Global mobile data traffic forecast update, 2009-2014,(white paper), 2010 http://cisco. biz/en, US/solutions/collateral/ns341/ns525/ns537/ns705/ns827/white_paper_c11520862. pdf.

[2] A. Vahdat, D. Becker, et al., Epidemic routing for partially connected ad hoc networks, Tech. rep., Technical Report CS-200006, Duke University (2000).

[3] U. Sadiq, M. Kumar, Proximol: Proximity and mobility estimation for efficient forwarding in opportunistic networks, in: 2011 Eighth IEEE International Conference on Mobile Ad-Hoc and Sensor Systems, IEEE, 2011, pp. 312-321.

[4] Y. Wang, S. Jain, M. Martonosi, K. Fall, Erasure-coding based routing for opportunistic networks, in: Proceedings of the 2005 ACM SIGCOMM workshop on Delay-tolerant networking, ACM, 2005, pp. 229-236.

[5] P. Santi, Routing in opportunistic networks, Mobility Models for Next Generation Wireless Networks: AD HOC, Vehicular and Mesh Networks (2012) 225-236.

[6] T. Camp, J. Boleng, V. Davies, A survey of mobility models for ad hoc network research, Wireless communications and mobile computing 2 (5) (2002) 483-502.

[7] M. Kim, D. Kotz, S. Kim, Extracting a mobility model from real user traces., in: INFOCOM, Vol. 6, 2006, pp. 1-13.

[8] L. Pajevic, G. Karlsson, A zero-dimensional mobility model for opportunistic networking, in: World of Wireless, Mobile and Multimedia Networks (WoWMoM), 2011 IEEE International Symposium on a, IEEE, 2011, pp. 1-6. 
[9] S. Qayyum, M. Shahriar, M. Kumar, S. K. Das, Pcv: Predicting contact volume for reliable and efficient data transfers in opportunistic networks, in: Local Computer Networks (LCN), 2013 IEEE 38th Conference on, IEEE, 2013, pp. 801-809.

[10] Y. Tseng, S. Kuo, H. Lee, C. Huang, Location tracking in a wireless sensor network by mobile agents and its data fusion strategies, in: Information Processing in Sensor Networks, Springer, 2003, pp. 554-554.

[11] R. Bajaj, S. Ranaweera, D. Agrawal, Gps: Location-tracking technology, Computer 35 (4) (2002) 92-94.

[12] S. Qayyum, U. Sadiq, M. Kumar, Meme: Real-time mobility estimation for mobile environments, in: Local Computer Networks (LCN), 2014 IEEE 39th Conference on, IEEE, 2014, pp. 133-141.

[13] L. Pelusi, A. Passarella, M. Conti, Opportunistic networking: data forwarding in disconnected mobile ad hoc networks, Communications Magazine, IEEE 44 (11) (2006) 134-141.

[14] T. Spyropoulos, K. Psounis, C. Raghavendra, Efficient routing in intermittently connected mobile networks: The single-copy case, IEEE/ACM Transactions on Networking (TON) 16 (1) (2008) 63-76.

[15] S. Menard, Applied logistic regression analysis, Vol. 106, Sage Publications, Incorporated, 2001.

[16] D. Hosmer, S. Lemeshow, Applied logistic regression, Vol. 354, WileyInterscience, 2000.

[17] Google, Sensormanager (Dec 2014).

URL http://developer.android.com/reference/android/ hardware/SensorManager.html

[18] I. Rhee, M. Shin, S. Hong, K. Lee, S. Kim, S. Chong, CRAWDAD trace, http://crawdad.cs.dartmouth.edu/ncsu/mobilitymodels/ GPS/NC_State_Fair(Jul. 2009). 
[19] K. Lee, S. Hong, S. Kim, I. Rhee, S. Chong, Slaw: A new mobility model for human walks, in: INFOCOM 2009, IEEE, IEEE, 2009, pp. 855-863.

[20] J. Ghosh, S. J. Philip, C. Qiao, Sociological orbit aware location approximation and routing (solar) in manet, Ad Hoc Netw. 5 (2) (2007) 189-209.

[21] S. C. Nelson, M. Bakht, R. Kravets, Encounter-based routing in dtns, in: INFOCOM 2009, IEEE, IEEE, 2009, pp. 846-854.

[22] J. Burgess, B. Gallagher, D. Jensen, B. N. Levine, Maxprop: Routing for vehiclebased disruption-tolerant networks., in: INFOCOM, Vol. 6, 2006, pp. 1-11.

[23] P. Hui, J. Crowcroft, E. Yoneki, Bubble rap: Social-based forwarding in delaytolerant networks, Mobile Computing, IEEE Transactions on 10 (11) (2011) $1576-1589$.

[24] E. M. Daly, M. Haahr, Social network analysis for routing in disconnected delaytolerant manets, in: Proceedings of the 8th ACM international symposium on Mobile ad hoc networking and computing, ACM, 2007, pp. 32-40.

[25] J. Ott, E. Hyytia, P. Lassila, T. Vaegs, J. Kangasharju, Floating content: Information sharing in urban areas, in: Pervasive Computing and Communications (PerCom), 2011 IEEE International Conference on, IEEE, 2011, pp. 136-146.

[26] C. Boldrini, M. Conti, A. Passarella, Contentplace: social-aware data dissemination in opportunistic networks, in: Proceedings of the 11th international symposium on Modeling, analysis and simulation of wireless and mobile systems, ACM, 2008, pp. 203-210.

[27] U. Sadiq, M. Kumar, A. Passarella, M. Conti, Modeling and simulation of service composition in opportunistic networks, in: Proceedings of the 14th ACM international conference on Modeling, analysis and simulation of wireless and mobile systems, ACM, 2011, pp. 159-168.

[28] J. Whitbeck, M. De Amorim, V. Conan, Plausible mobility: inferring movement from contacts, arXiv preprint arXiv:1001.3673. 